such cases. Accurate diagnosis and hemodynamic assessments by cardiac catheterization were necessary before surgical repair. Surgical repair was successfully done by division of the anomalous PV without cardiopulmonary bypass. Our case showed the possibility of coexistence of normal and anomalous PVs in the common situation of PAPVC of the right upper PV. Surgeons must keep in mind this rare anomaly and its repair.

\section{Conclusion}

We reported surgical repair of PAPVC of the right upper PV, which connected both to the SVC and to the LA, showing retrograde blood flow from the LA to the SVC.

\section{References}

1. Bahnson HT, Spencer FC, Neill CA. Surgical treatment of thirty-five cases of drainage of pulmonary veins to the right side of the heart. J Thorac Surg. 1958;36:777-9.

2. Lewis FJ. High defects of the atrium. J Thorac Cardiovasc Surg. 1958;36:1-5.

3. Swan HJ, Kirklin JW, Becu LM, Wood EH. Anomalous connection of right pulmonary veins to superior vena cava with intra-atrial communications: hemodynamic data in eight cases. Circulation. 1957;16:5466.

4. Beck C, Wessel A, Yelbuz TM, Bertram H. Unusual case of anomalous pulmonary venous return with left atrial to systemic venous shunt Circulation. 2006;113:e840-1.

\title{
Biventricular mechanical assist for complex, acute post-infarction ventricular septal defect
}

\author{
Chittoor B. Sai-Sudhakar, MBBS, FRCS, Michael S. Firstenberg, MD, and Benjamin Sun, MD, Columbus, Ohio
}

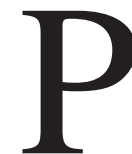

ost-infarction ventricular septal defects (PI-VSDs) represent a catastrophic complication after acute myocardial infarctions. Despite advances in surgical and postoperative management, operative mortality is still $10 \%$ to $60 \% .{ }^{1}$ Furthermore, little has been written describing the options in managing the severe and often biventricular failure that is associated with PI-VSD and that is a significant factor influencing postoperative morbidity and mortality. We describe a patient with complex PI-VSD who, despite appropriate and prompt surgical management, required biventricular mechanical assist devices (biVADs) as a bridge to recovery.

\section{Case Report}

A 53-year-old man was admitted to an outside hospital with vague abdominal symptoms and a presumed diagnosis of acute cholecystitis. Severe hypotension and a new murmur shortly developed. Echocardiography revealed a large posteroinferior PI-VSD. After the patient was urgently transferred to our medical center, cardiac catheterization revealed a complete right coronary artery occlusion with critical left anterior descending coronary artery and ramus diseases. Because the patient was in profound cardiogenic shock requiring multiple high-dose pressors and intraaortic counter pul-

\footnotetext{
From the Department of Cardiothoracic Surgery, The Ohio State University, Columbus, Ohio.

Received for publication May 18, 2006; accepted for publication June 27, 2006.

Address for reprints: Michael S. Firstenberg, MD, Division of Cardiothoracic Surgery, N846 Doan Hall, 410 W. 10th Avenue, The Ohio State University, Columbus, OH 43210 (E-mail: msfirst@gmail.com).

J Thorac Cardiovasc Surg 2006;132:1238-9

$0022-5223 / \$ 32.00$

Copyright $\odot 2006$ by The American Association for Thoracic Surgery doi:10.1016/j.jtcvs.2006.06.037
}

sation, he was taken urgently to the operating room. Intraoperatively, the patient's heart was diffusely erythematous, and there was a large inferior wall infarct, an akinetic right ventricle, and severe mitral regurgitation from a ruptured papillary muscle. Transesophageal echocardiology demonstrated a $4 \times 4-\mathrm{cm}$ posteroinferior VSD extending to the annulus of the mitral valve. After standard bicaval cannulation, cardiopulmonary bypass, and antegrade and retrograde cardioplegic arrest, an incision was made parallel to the posterior descending artery. The defect was closed with a large bovine pericardial patch with pledgeted sutures through healthy-appearing tissue. Coronary artery bypass grafting was performed in the diseased vessels. A mechanical mitral valve was inserted using standard techniques. Secondary to severe intraoperative right ventricular failure, a right ventricular assist device (Abiomed 5000, Abiomed Inc, Danvers, Mass) was placed. However, with increasing right ventricular assist device flows, left to right shunting across a residual VSD increased. Because of the perceived technical and physiologic difficulties associated with attempts at closure, combined with worsening left ventricular function, a left ventricular assist device (Abiomed 5000) was placed. The patient was stabilized and weaned from bypass. His initial intensive care unit course was unremarkable as he was rapidly extubated and weaned from pharmacologic support. After several months of aggressive rehabilitation, the patient underwent explantation of his biVAD and residual VSD closure. The patient tolerated this procedure well, and after pharmacologic optimization of his chronic heart failure he was transferred to an outpatient rehabilitation facility. At 18 months post-infarction, the patient was well.

\section{Discussion}

PI-VSDs complicate up to $0.02 \%$ of myocardial infarctions. ${ }^{2}$ Despite advances in the surgical care of these moribund patients, operative mortality still approaches $50 \%$ with major risks, including cardiogenic shock, renal failure, right and/or left ventricular 
failure, size of VSD, posterior/inferior locations, and residual VSD. ${ }^{3}$ Management consists of VSD closure, coronary artery bypass grafting as needed, and aggressive postcardiotomy and end-organ support. Residual shunts are found in up to $40 \%$ of patients. Operative repair is associated with mortality greater than $60 \%,{ }^{4}$ which has prompted interest in percutaneous closure, but the role of these procedures is still undefined. ${ }^{5}$ In our case, the implantation of a biVAD served as a bridge to recovery. By minimizing associated postoperative end-organ damage with cardiac support, we allowed for physiologic recovery so the residual VSD could be successfully closed and the impact of the massive myocardial infarction and associated heart failure could be minimized. Limitations include the need for multiple procedures (ie, device weaning/explant), aggressive anticoagulation in a high-risk patient, and meticulous follow-up.

Right ventricular support is critical after the acute volume and pressure overload of a PI-VSD. Recovery may be unpredictable and prolonged, necessitating long-term biventricular support. Short-term univentricular options, such as intraaortic balloon pumps, extracorporeal membrane oxygenation, or left ventricular assist devices, are limited in right ventricle failure and residual shunting. A total artificial heart precludes native cardiac recovery and obligates transplantation; nevertheless, this may be an option with the appropriate resources and experience.

\section{Conclusion}

Biventricular mechanical support, as demonstrated in this complex case, should be considered a useful tool as a bridge to recovery in patients with PI-VSDs.

\section{References}

1. Cummings RG, Califf R, Jones RN, Reimer KA, King YH, Lowe JE. Correlates of survival in patients with postinfarction ventricular septal defect. Ann Thorac Surg. 1989;47:824-30 (update: 1997;63:1508-9).

2. Crenshaw BS, Granger CB, Birnbaum Y, et al. Risk factors, angiographic patterns and outcomes in patients with ventricular septal defect complicating acute myocardial infarction. GUSTO-1 (Global Utilization of Streptokinase and TPA for Occluded Coronary Arteries). Trial Investigators. Circulation. 2000;101:27-32.

3. Labrousse L, Choukroun E, Chevalier JM, et al. Surgery for postinfarction ventricular septal defect (VSD): risk factors for hospital death and long term results. Euro J Cardiothorac Surg. 2002;21: 725-31.

4. Jeppsson A, Liden H, Johnsson P, Hartford M, Radegran K. Surgical repair of post infarction ventricular septal defects: a national experience. Euro J Cardiothorac Surg. 2005;27:216-21.

5. Pienvichit P, Piemonte TC. Percutaneous closure of postmyocardial infarction ventricular septal defect with the CardioSEAL septal occluder implant. Catheter Cardiovasc Interv. 2001;54:490-4.

\title{
Atypical presentation of extralobar sequestration with absence of pericardium in an adult
}

\author{
Gourab Datta, MB, ChB, Jeymi Tambiah, MS, FRCS, Sheila Rankin, FRCR, Amanda Herbert, FRCPath, \\ and Loïc Lang-Lazdunski, MD, PhD, FRCS, London, United Kingdom
}

$\mathrm{E}$ xtralobar sequestration (ELS) is a rare congenital lesion of the lung. We report a case of ELS with concurrent absence of left pericardium presenting in an adult as an anterior mediastinal mass.

\section{Clinical Summary}

A 32-year-old man presented with a 3-week history of anterior chest discomfort. He denied any weight loss, night sweats, or fever. Clinical examination was unremarkable. Chest radiography and

From the Department of Thoracic Surgery, Guy's Hospital, London, United Kingdom.

Received for publication April 1, 2006; revisions received April 3, 2006; accepted for publication May 17, 2006.

Address for reprints: Loïc Lang-Lazdunski, MD, PhD, FRCS, Department of Thoracic Surgery, Guy's Hospital, St Thomas St, London SE1 9RT, United Kingdom (E-mail: loic.lang-lazdunski@gstt.nhs.uk).

J Thorac Cardiovasc Surg 2006;132:1239-40

$0022-5223 / \$ 32.00$

Copyright () 2006 by The American Association for Thoracic Surgery doi:10.1016/j.jtcvs.2006.05.054 computed tomography $(\mathrm{CT})$ revealed a cystic mass in the left anterior mediastinum $(80 \times 80 \times 70 \mathrm{~mm})$ in close proximity to the pulmonary trunk (Figure 1). Tumor markers, including $\alpha$-fetoprotein, $\beta$-human chorionic gonadotropin, and lactate dehydrogenase, were normal. A bronchogenic cyst or a thymic cyst was suspected. A CT-guided biopsy was performed but was inconclusive. The patient was referred to our department for biopsy or excision of the lesion.

We approached the mass through an anterior minithoracotomy via the left second intercostal space. An initial noteworthy finding was the absence of pericardium. A large cystic lesion with a smooth grayish surface was visualized, and amorphous brownish liquid was aspirated from it. Macroscopically, the lesion had the appearance of a bronchogenic cyst and was adherent to the mediastinum and pulmonary hilum. The mass was dissected from surrounding structures progressively by using diathermy and ligaclips, but extraction resulted in massive bleeding. The incision was enlarged, and a clamp was applied to the origin of the left pulmonary artery (LPA). The bleeding source was identified as a small vessel $(2 \mathrm{~mm})$ originating from the proximal LPA. It was controlled with a single purse-string suture. The mass was completely excised. The patient recovered uneventfully and was discharged on day 5 . 